\title{
MUSÉES ARCHÉOLOGIQUES D'ALEXANDRIE ET DU DELTA DU NIL
}

\author{
MARIE-CÉCILE BRUWIER \\ marie-cecile.bruwier@musee-mariemont.be \\ MusÉE RoYAL de MARIEMONT - UNIVERSITÉ CATHOLIQUE DE LOUVAIN \\ http://dx.doi.org/10.25267/Riparia_sup.2018.11.13
}

Depuis sa création au XIX ${ }^{\mathrm{e}}$ siècle, le Service des Antiquités, appelé ensuite Conseil Suprême des Antiquités de l'Égypte $(\mathrm{CSA})^{1}$, gère, protège et restaure, dans la mesure du possible, les monuments et antiquités qui ont plus de cent ans. Jusqu'en 2011, cette institution dépendait du Ministère de la Culture. Aujourd'hui, elle est devenue Ministère des Antiquités (MA). Elle organise et prend en charge la plupart des musées du pays.

Outre le MA, d'autres instances gèrent des institutions muséales majeures. Tel est le cas, par exemple, de l'un des premiers musées du pays, le Musée d'Ethnographie fondé en 1898 au sein de la Société de Géographie d'Égypte créée quant à elle en 1875. À ce moment-là, existent déjà le musée des antiquités pharaoniques ou musée égyptien depuis 1857-1858, le Musée d'Art islamique dès 1880 et le musée grécoromain d'Alexandrie datant de $1892^{2}$. Signalons aussi le Musée de géologie ouvert en 1904, le Musée de l'Agriculture fondé en 1930 qui relève du Ministère de l'Agriculture. Quant au Musée national égyptien des chemins de fer, construit en 1932 dans la gare Ramsès, il dépend du Ministère des Transports ${ }^{3}$.

À la suite du tremblement de terre qui a affecté la région du Caire en 1992, divers projets émanant du CSA et d'autres organismes ont vu le jour, destinés à préserver et à valoriser l'héritage culturel de l'Égypte pharaonique, gréco-romaine, copte et musulmane, tout autant que le patrimoine immatériel contemporain. L'acuité de la protection du patrimoine a engendré la création d'ECHO (Egyptian Cultural Heritage Organisation ${ }^{4}$.

Aujourd'hui en Égypte, la question de la place du patrimoine culturel se pose plus crucialement encore depuis la révolution du 25 janvier 2011. L'une des conséquences de cet événement historique est la baisse du tourisme et le manque de rentrées financières qui en découle. En même temps, des manifestations occasionnelles d'hostilité vis-à-vis du patrimoine (pillage du musée du Caire, de magasins de sites archéologiques $^{5}$, du musée de Mallawi ${ }^{6}$, proposition de fatwa pour la destruction des

${ }^{1}$ F. HAIKAL, «Egypt's Past Regenerated by its own people », in S. MACDONALD, M. RICE (éd.), Consuming Ancient Egypt, Londres, Institute of Archaeology, University College, 2003, p. 123-138.

2 E. Perrin, «Le Musée d'Ethnographie de la Société de Géographie d’Égypte», in Gradbiva, 2 (2005), p. 5-29 et particulièrement, p. 6.

${ }^{3}$ F. HASSAN, «Les musées égyptiens oubliés », in Paysages du Patrimoine en Égypte (Museum International, 225-226, mai 2005), p. 42-48.

${ }^{4}$ http://www.e-c-h-o.org/mandate.php. F.A. Hassan, L. S. Owens, A. DE TrafFord, G. J. TAssie \& J. Van Wetering (éds.), Managing Egypt's cultural heritage: Proceedings of the First Egyptian Cultural Heritage: Egyptian Cultural Heritage Management, Londres, Golden House Publications, 2009.

${ }^{5} \mathrm{http}: / /$ www.lepoint.fr/actu-science/le-pillage-du-patrimoine-egyptien-02-02-2011134568 59.php 
pyramides et du sphinx de Giza ${ }^{7}$ ) ont intensifié l'importance de la préservation de ce patrimoine. Un an après la révolution, les actions de l'International Council of Museums (ICOM) visant à protéger le patrimoine culturel des vols dans les musées et d'objets non inventoriés dans les sites archéologiques ont abouti à la publication d'une Liste Rouge des biens culturels égyptiens en péril ${ }^{8}$.

Parmi diverses réponses égyptiennes figure notamment la création en 2000 de CULTNAT (Center for Documentation of Cultural and Natural Heritage) ${ }^{9}$. Conçu en vue de la sensibilisation au patrimoine par le biais de formations, d'expositions et de publications, ce centre national d'interprétation soutenu par la Bibliotheca Alexandrina documente les différents aspects du patrimoine naturel et culturel.

En 2005, le CSA a programmé la construction ou la rénovation de trente-trois musées ${ }^{10}$. Cette sensibilisation au Patrimoine a conduit à aménager plusieurs sites clés du pays en y construisant des musées locaux dédiés aux objets mis au jour sur place ${ }^{11}$. Plusieurs nouveaux musées ont été créés, en particulier à Assouan et à Alexandrie. D’autres ont été rénovés, modernisés et plusieurs grands projets muséaux sont en cours d'élaboration, tels le Grand Musée égyptien (GEM) à proximité des pyramides du Plateau de Giza ou le Musée National de la Civilisation Égyptienne (NMEC) sur le site archéologique d'El-Fustat. Parmi les créations récentes, citons les musées Imhotep (Saqqara), d'El-Arish ou Sobek (Kom Ombo). Divers musées ont été rénovés, tels les musées d'art islamique au Caire, de Kom el-Ouchim et de Mallawi ${ }^{12}$ déjà cité. La rénovation du musée gréco-romain d'Alexandrie est toujours en cours.

\section{Défis de la conservation du patrimoine du Delta du Nil}

La compréhension de l'histoire du Delta s'avère fondamentale pour la connaissance de la civilisation égyptienne. La région, multiculturelle pendant des siècles, a vu la cohabitation d'Égyptiens avec des Libyens, des Phéniciens, des Grecs et des Romains...

Plusieurs obstacles compliquent la préservation du patrimoine qu'il recèle. Les vestiges archéologiques sont en particulier menacés par la montée des eaux, la pluviométrie élevée dans le Delta et l'expansion urbaine. Comme l'explique $\mathrm{Ph}$. Brissaud dans le cas de Tanis ${ }^{13}$, le site est très fragile et sujet à une érosion importante du fait de ses dimensions et de son altitude élevée. Le sous-sol fort salé altère

${ }^{6} \mathrm{http}: / / \mathrm{www}$.unesco.org/new/fr/culture/themes/illicit-traffic-of-culturalproperty/emergency-actions/egypt/warning-looting-of-the-malawi-national-museum

${ }_{7}$ http://www.express.be/joker/fr/world/les-pyramides-egyptiennes-sont-elles-menaceesde-destruction $/ 172250 . h$ tm

${ }^{8}$ http://icom.museum/resources/red-lists-database/red-list/egypt

${ }^{9}$ F. SALEH, H. N. BARAKAT, « Le village planétaire du patrimoine : la contribution du Centre de documentation sur le patrimoine culturel et naturel (CULTNAT) », in Paysages du patrimoine en Égypte: Museum international, 225-226 (57, 1-2) (2005), p. 73-78. www.cultnat.org; http://en.wikipedia.org/wiki/Center for Documentation of Cultural and Natural Herita ge

${ }^{10}$ Z. HAWASS, "Une nouvelle ère pour les musées égyptiens", in Paysages du patrimoine en Égypte: Museum international, 225-226 (57, 1-2) (2005), p. 7-23.

11 M.-C. BRUWIER \& Fr. MAIRESSE, «À qui appartient le patrimoine égyptien? La mondialisation de la culture pharaonique et l'histoire des musées en Égypte », in Patrimoine et mondialisation, Paris, L'Harmattan, 2008, p. 35-56.

12 http://www.nileinternational.net/fr/?p=53159

${ }^{13} \mathrm{Ph}$. BRISSAUD, «Tanis : protection du patrimoine et recherche archéologique à la lumière des derniers travaux ", in Egyptology at the Dawn of the Twenty-first Century: Proceedings of the Eighth International Congress of Egyptologists, Le Caire, 2000, vol. 3, p. 143. 
considérablement les antiquités quel que soit leur matériau. Consciente des dangers encourus par ce patrimoine, l'Égypte a lancé un appel aux archéologues pour qu'ils concentrent leurs recherches archéologiques dans le Delta.

Alexandrie présente un cas particulier. Les occupations successives de l'Antiquité à l'époque contemporaine ont recouvert ce qui subsistait de la cité antique. L'étude du patrimoine enfoui ne peut s'y faire que par des fouilles de sauvetage exécutées rapidement. Ainsi par exemple, la Necropolis, la «ville des morts » ainsi nommée dans l'Antiquité par le géographe Strabon, a été retrouvée en 1997 à l'occasion des travaux de construction d'une autoroute urbaine à Gabbari. La découverte conduit alors les autorités égyptiennes à confier le chantier archéologique au Centre d'études alexandrines. Seule, une petite partie de cet immense cimetière utilisé du $\mathrm{III}^{\mathrm{e}}$ siècle avant J.-C. au VII ${ }^{\text {e }}$ siècle après J.-C. a pu être étudiée avant l'achèvement de la voie contemporaine.

Ajoutons à cela le phénomène de subsidence ${ }^{14}$ qui affecte la côte méditerranéenne. À Alexandrie, l'affaissement du littoral aux IV et $\mathrm{V}^{\mathrm{e}}$ siècles de notre ère a englouti sous les eaux le quartier où se trouvaient dans l'Antiquité les installations portuaires et les bâtiments royaux. Cette même zone a été partiellement remblayée aux XIX ${ }^{\mathrm{e}}-\mathrm{XX}^{\mathrm{e}}$ par la construction d'une corniche et l'aménagement de ports modernes. De même près d'Aboukir (Canope), l'antique Thonis (Herakleion) se trouve aujourd'hui à plusieurs km au large. Comme pour les célèbres monuments de l'Alexandrie antique, les vestiges de cette cité ne sont plus connus que par les fouilles sous-marines qui y sont conduites et qui ont été présentées au public tant à Bonn, Berlin, Madrid, Paris ou Londres lors d'expositions temporaires au titre révélateur sur les cités et trésors engloutis.

Depuis 1998, le souci de protéger, de conserver et d'aménager le patrimoine culturel subaquatique $^{15}$ alexandrin a généré un projet de musée sous-marin à l'initiative des autorités égyptiennes et de l'UNESCO. Ce musée partiellement immergé exposerait le patrimoine in situ, conformément aux principes éthiques et scientifiques de la Convention de 2001 de l'UNESCO sur la protection du patrimoine culturel subaquatique. Mis de côté après la révolution de 2011, le projet ranimé en septembre 2015 présente divers objectifs : rendre les antiquités sous-marines accessibles au public, relancer le tourisme ${ }^{16}$ mais aussi protéger le site archéologique de la pollution, des ancres des bateaux de pêche et du pillage.

\section{Principaux musées du Delta}

Plusieurs sources ${ }^{17}$ aident à dresser un premier inventaire des musées actuels du Delta. Le site du Ministère des Antiquités ${ }^{18}$ présente une liste de musées sous son autorité. Celle qui est publiée en 2005 annonce le programme de construction et de rénovation de musées ${ }^{19}$. Deux autres sites complètent ce tableau ${ }^{20}$ du paysage muséal égyptien.

\footnotetext{
${ }^{14}$ http://www.cealex.org/sitecealex/activites/etudes SUBSIDENCE F.HTM

15 http://www.unesco.org/new/fr/culture/themes/underwater-cultural-heritage/museumsand-tourism/alexandria-museum-project/

${ }^{16}$ http://www.archibat.com/blog/un-musee-sous-marin-pour-la-ville-dalexandrie/ ;

http://www.atlantico.fr/decryptage/projet-pharaonique-musee-subaquatique-qui-pourraitrelancer-tourisme-en-egypte-dans-annees-venir-2518408.html

17 Voir notamment International Directory of Arts 2016, 40e edition, octobre 2015.

${ }^{18} \mathrm{http}: / /$ www.sca-egypt.org/eng/MUS List.htm

${ }^{19}$ Z. HAWASS, op. cit., p. 21-23.
} 
Les musées du Delta font l'objet d'un projet scientifique issu d'une collaboration égypto-allemande. Museums in the Nile Delta (M.I.N.), mis sur pied par le Prof. Mohammed I. Bakr et le Prof. Helmut Brandl de l'Université Humbolt à Berlin, a été fondé pour faire connaitre les collections archéologiques de musées peu connus et donc peu visités par les touristes. À cette fin, M.I.N. publie des catalogues scientifiques dans lesquelles se trouvent les informations essentielles sur le contexte des découvertes des archéologues égyptiens, par exemple, à Tanis ou Bubastis ${ }^{21}$.

\section{Musées d'antiquités}

La longue histoire d'Alexandrie explique le nombre important de ses musées. Parmi les plus anciens d'Égypte figure le Musée gréco-romain ${ }^{22}$, inauguré en 1892. La nécessité de conserver les découvertes archéologiques était apparue après 1866 à la suite des fouilles conduites par Mahmoud bey dit el-Falaki (1815-1885). Avant sa fermeture pour rénovation en 2005, il présentait des monuments et des objets provenant de toute la Basse-Égypte et dont la plupart relèvent du monde grécoromain. L'espace consacré à l'Égypte pharaonique se limitait à une salle et à une partie du jardin.

$\mathrm{Au}$ cours de la dernière décennie, trois nouveaux musées archéologiques ont été inaugurés. Celui de la Bibliotheca Alexandrina, ouverte en 2002, présente des objets des époques hellénistique, romaine et byzantine exhumés lors des travaux de fondation de la Bibliothèque. Centrées sur Alexandrie et la période ptolémaïque, les collections comportent aussi des pièces des fouilles sous-marines du port Est de la ville et de l'île Nelson. Les cultures pharaonique, copte et musulmane y sont aussi représentées.

Tel est également le cas au Musée national d'Alexandrie aménagé depuis 2003 dans un ancien consulat. Des œuvres majeures du passé pharaonique, copte et islamique de l'Égypte y côtoient des monuments issus des fouilles sous-marines d'Alexandrie.

Quant au Musée archéologique du Patriarcat d'Alexandrie et de toute l'Afrique, il regroupe également des objets de l'époque pharaonique, hellénistique, byzantine et islamique.

Créé en 1945, un demi-siècle après le Musée gréco-romain, le Musée archéologique de la Faculté des Lettres, chronologiquement la seconde institution muséale d'Alexandrie, ne relève pas du CSA $^{23}$. Il est le plus ancien musée universitaire d'Égypte ${ }^{24}$, destiné à conserver le matériel archéologique mis au jour lors des fouilles conduites par l'Université en Égypte et en Nubie. Il constitue une sorte de « laboratoire » pour les chercheurs et les étudiants en archéologie et en histoire de l'art

${ }^{20} \mathrm{https} / / /$ en.wikipedia.org/wiki/List of museums in Egypt ;

http://www.desheret.org/egypt.html

${ }_{21}^{21}$ M. I. BAKR, H. BRANDL, F. KALlONIATIS (éds), Egyptian Antiquities from Kufur Nigm and Bubastis in Museums in the Nile Delta, 1, Le Caire-Berlin, Opaion, 2010 ; ID., Egyptian Antiquities from the Eastern Nile Delta, in Museums in the Nile Delta, 2, Le Caire-Berlin, Opaion, 2014.

${ }^{22}$ Sur la muséologie et la muséographie du Musée gréco-romain d'Alexandrie, voir Ph. JOCKEY, «Le Musée gréco-romain d'Alexandrie ou les musées imaginaires d'Evaristo Breccia », in S. BASCH, J.-Y. EMPEREUR, Alexandria ad Europam (Études Alexandrines 14 (2007), p. 25-50.

23 J.-Y. Empereur, M. Haggag, "The Museum of the Faculty of Arts, Alexandria University », in H. M. S. ABDEL NABY, D. DiXneuf, Islamic Pottery, 1: Catalogue of the Faculty of Arts Museum Alexandria University in Études Alexandrines 28, 2013, p. 9-15.

${ }^{24}$ M. HAGGAG, "The Educational Museum of the Faculty of Arts », in K. SAVvOpoulos (éd.), Proceedings of the First Hellenistic Studies Workshop, Alexandria, 12-18 july 2009, Alexandrie, 2010, p. 110-117. 
qui peuvent étudier les objets de près et disposer d'ensembles représentatifs des productions égyptiennes dans les arts et les techniques. Elles couvrent une vaste période chronologique, de la préhistoire à l'Égypte pharaonique, gréco-romaine, copte et musulmane. Une collaboration franco-égyptienne lie la Faculté des Lettres au Centre d'Études alexandrines pour la publication du catalogue des collections. Le premier volume consacré à la céramique islamique a été publié ${ }^{25}$; un second volume dédié aux objets d'époque pharaonique paraitra prochainement avec le concours du Musée royal de Mariemont (Belgique).

Le Delta compte d'autres musées archéologiques majeurs. Le Musée de Tanta, datant de 1913, contient notamment des œuvres provenant de Saïs, Naucratis et Bouto.

Créé en 1932, dans un bâtiment dont l'architecture s'inspire d'un temple antique, le Musée d'Ismailia ${ }^{26}$ implanté dans un jardin conserve des objets d'époque pharaonique, ptolémaïque et romaine.

Le Musée national de Charqeyya ${ }^{27}$ (Herriat Raznah) ${ }^{28}$, ouvert en 1973, a malheureusement été fermé en 2006. Il présentait un grand nombre d'antiquités de toutes les périodes de l'histoire pharaonique, de l'époque gréco-romaine et du début de l'Islam ainsi que des objets étrangers importés en Égypte dans l'Antiquité. Leur provenance et le contexte de leur découverte est assuré dans la mesure où tous les objets ont été trouvés et enregistrés par des archéologues égyptiens dans les années 1960 et 1970. Aujourd'hui, de nombreuses pièces ont été transférées, notamment au Musée de site de Tell Basta (Bubastis) ouvert en 2016.

Quant au Musée national de Port Saïd ${ }^{29}$, fondé en 1987, il est entouré d'un jardin où l'on retrouve les stèles autrefois déposées dans le jardin d'Ismailia. Son contenu couvre toute l'histoire de l'Égypte, de la préhistoire à l'époque moderne et beaucoup d'objets proviennent d'un dépôt du musée du Caire.

Le Musée de l'Université de Zagazig ${ }^{30}$ ouvert en 1992 à l'initiative du Prof. Mohammed I. Bakr ${ }^{31}$ conserve un grand nombre d'antiquités provenant notamment de Tell Basta et de Kufur Nigm et mises au jour depuis 1978 par la mission archéologique de l'Université. L'accroissement considérable du nombre des découvertes a nécessité le déménagement du musée dans un bâtiment plus grand.

Le Musée de la civilisation planifié à Khafr el-Sheikh ${ }^{32}$ pour évoquer la civilisation égyptienne de la préhistoire à nos jours, ouvert en 2005, a connu quelques turbulences ${ }^{33}$.

Le Musée de Suez consacré aux vestiges archéologiques provenant des fouilles dans la région a été inauguré en $2012^{34}$. Le bâtiment à l'architecture moderne se situe dans

\footnotetext{
25 Voir note 25.

26 Fr. GOURDON, «Les trésors cachés des petits musées d'Égypte», 2e partie, in Toutankhamon Magazine, 15 (juin/juillet 2004), p. 61.

${ }^{27}$ ID., «Les trésors cachés des petits musées d'Égypte », 1 ìre partie, in Toutankhamon Magazine, 14 (avril/mai, 2004), p. 63-64.

${ }^{28} \mathrm{http}$ //project-min.de/home/english/herriat $\% 20$ razna en.html ; voir note 23 .

${ }^{29}$ Fr. GOURDON, op. cit., $2^{\mathrm{e}}$ partie, p. 62.

${ }^{30}$ http://english.zu.edu.eg/Musium.aspx; Fr. GOURDON, op. cit., 1 ère partie, p. 64.

31 Voir note 23.

32 http://www.sca-egypt.org/eng/MUS List.htm

33 http://alamirkamalfarag.com/show news.php?id=1178;

http://helloegy.net/show news.php?id $=1105$
} 
un jardin présentant des œuvres antiques ; un bassin accueille la reconstitution d'un bateau d'époque pharaonique.

Parmi les nouveaux musées de site annoncés en $2005^{35}$, figure celui de San el-Hagar (Tanis). Un musée est en construction sur la route entre Tell El-Dab'a (Avaris) et San El-Hagar. Une source mentionne un musée en construction à l'Université de Munufiyya pour abriter la collection de la Faculté de Tourisme et d'Hôtellerie ${ }^{36}$.

\section{Autres musées}

À Alexandrie, s’inscrivant dans la succession de la bibliothèque et du Mouseion de l'Antiquité, la Bibliotheca Alexandrina compte également un musée des manuscrits, un Musée Shadi Abdel Salam, un Musée Sadate visant la documentation de l'Égypte moderne $^{37}$, un Planétarium, et un Musée des Sciences. Signalons aussi le Musée maritime, le Musée des Bijoux de la famille royale (juin 2005) ${ }^{38}$ et le Musée Constantin Cavafy $^{39}$.

La ville côtière de Rashid (Rosette) a vu de nombreuses restaurations de bâtiments par le SCA dans les années 2000, transformant la ville en véritable musée architectural de l'époque ottomane.

À Port Saïd, méritent le détour un Musée militaire, ouvert en 1964 pour célébrer la victoire de 1956 contre la France, la Grande-Bretagne et Israël et le Musée d'Art moderne, ouvert en 1995.

Quant au Musée de Denshway, il commémore les évènements qui s'y sont déroulés ${ }^{40}$ en 1906, lors de la révolte paysanne contre la présence britannique.

Le projet du Musée du Canal de Suez ${ }^{41}$ à Ismailia, lancé en 2013, vise à promouvoir l'histoire du Canal, l'une des principales sources de devises étrangères pour l'Égypte. Citons pour clore cet inventaire Le Pont de la Civilisation ${ }^{42}$ à Damiette qui constitue un exemple unique de conservation du patrimoine industriel en Égypte. Quatre sections du pont d'Imbaba au Caire ont été récupérées et transportées en 1927 à Damiette ; lors du remplacement du pont de Damiette en 2003, ces éléments historiques étaient destinés à la refonte; le gouverneur en a sauvé la partie mobile pour en faire une extension de la Mubarak Library, ouverte en 2009.

Depuis une vingtaine d'années, le rôle majeur des musées en matière de protection, de conservation et d'interprétation du patrimoine égyptien est clairement apparu.

\footnotetext{
${ }^{34}$ http://hebdo.ahram.org.eg/NewsContent/1045/32/97/7201/Le-Mus\%C3\%A9e-de-

$\underline{\text { Suez-voit-enfin-le-jour.aspx }}$

35 Z. HAWASS, op. cit., p. 19.

36 http://www.desheret.org/egypt.html

${ }^{37} \mathrm{http://www.bibalex.org/fr/center/details/sadatmuseum}$

${ }^{38}$ http://english.ahram.org.eg/NewsContent/9/0/113425/Heritage/0/Egypts-royaljewellery-now-on-display-at-Alexandri.aspx;

http://www.egyptianmuseums.net/html/alexandria s jewellery museum.html

${ }^{39} \mathrm{http}$ // hfc-worldwide.org/museums/cavafy-museum/

http://www.fodors.com/world/africa-and-middle-east/egypt/alexandria/things-todo/sights/reviews/the-cavafy-museum-470406

${ }^{40} \mathrm{http}: / /$ www.travelcenturies.com/Depdetails.aspx?ID=143\&Name=Denshway Museum, Al-Minufiyah

${ }^{41}$ https://www.rtbf.be/culture/arts/detail l-egypte-construit-un-musee-dedie-au-canal-desuez?id $=8172809$

42 http://cairobserver.com/post/12492028959/old-imbaba-bridge-1890\#.WIt8FfnhDIU
} 
Estimant que la clef stratégique d'éducation et de conservation se trouve dans les musées, le CSA et le Ministère de la Culture initient de nouvelles politiques muséales ${ }^{43}$. Le MA publie une lettre d'information montrant l'avancement des projets et mettant ses actions en lumière ${ }^{44}$. L'urgence de la formation de conservateurs de musées à la muséologie et à la muséographie a incité l'UNESCO ${ }^{45}$ et l'Université française d'Égypte $^{46}$ à créer des programmes spécialisés en la matière. Nombreux sont donc les acteurs professionnels conscients du caractère essentiel de la médiation patrimoniale. Gageons que les moyens leur seront accordés, dans l'Égypte en devenir, pour promouvoir le passé afin de préserver l'avenir de son patrimoine.

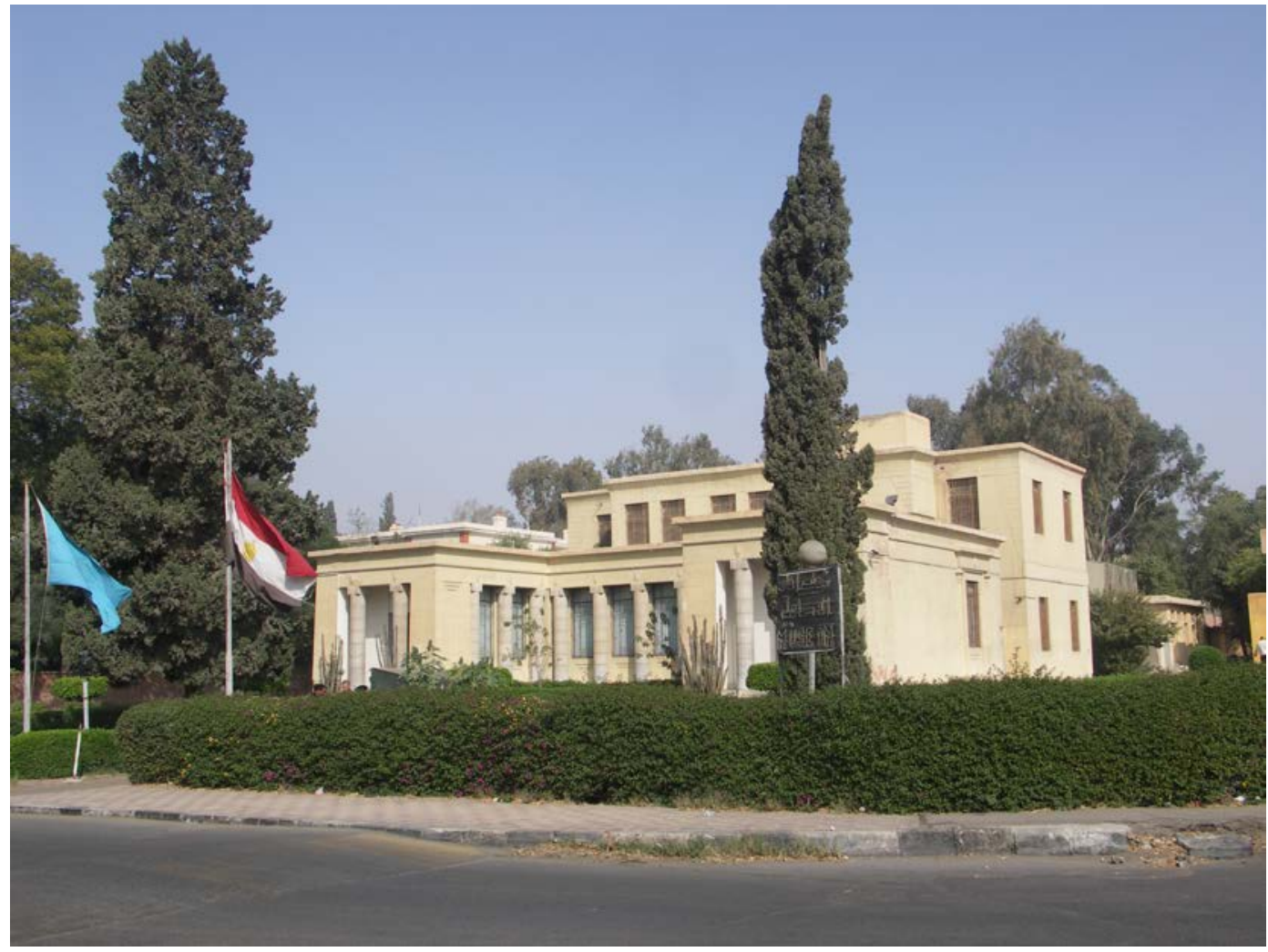

Musée d'Ismaïlia (photo V. EUVERTE).

43 R. BALIGH, "Museum Education in Egypt and the World", in Bulletin of the Egyptian Museum 2 (2005), p. 23-28.

${ }^{44}$ http://www.egyptologyforum.org/MOA/MOA.html

${ }^{45}$ https://www.ungm.org/Notices/Item.aspx?Id=21378

${ }_{46}$ Master Degree in Cultural Heritage Management (Heritage for a better future); voir www.ufe.edu.eg. 




Salle du Musée d'Ismaillia (photo V. EUVERTE).

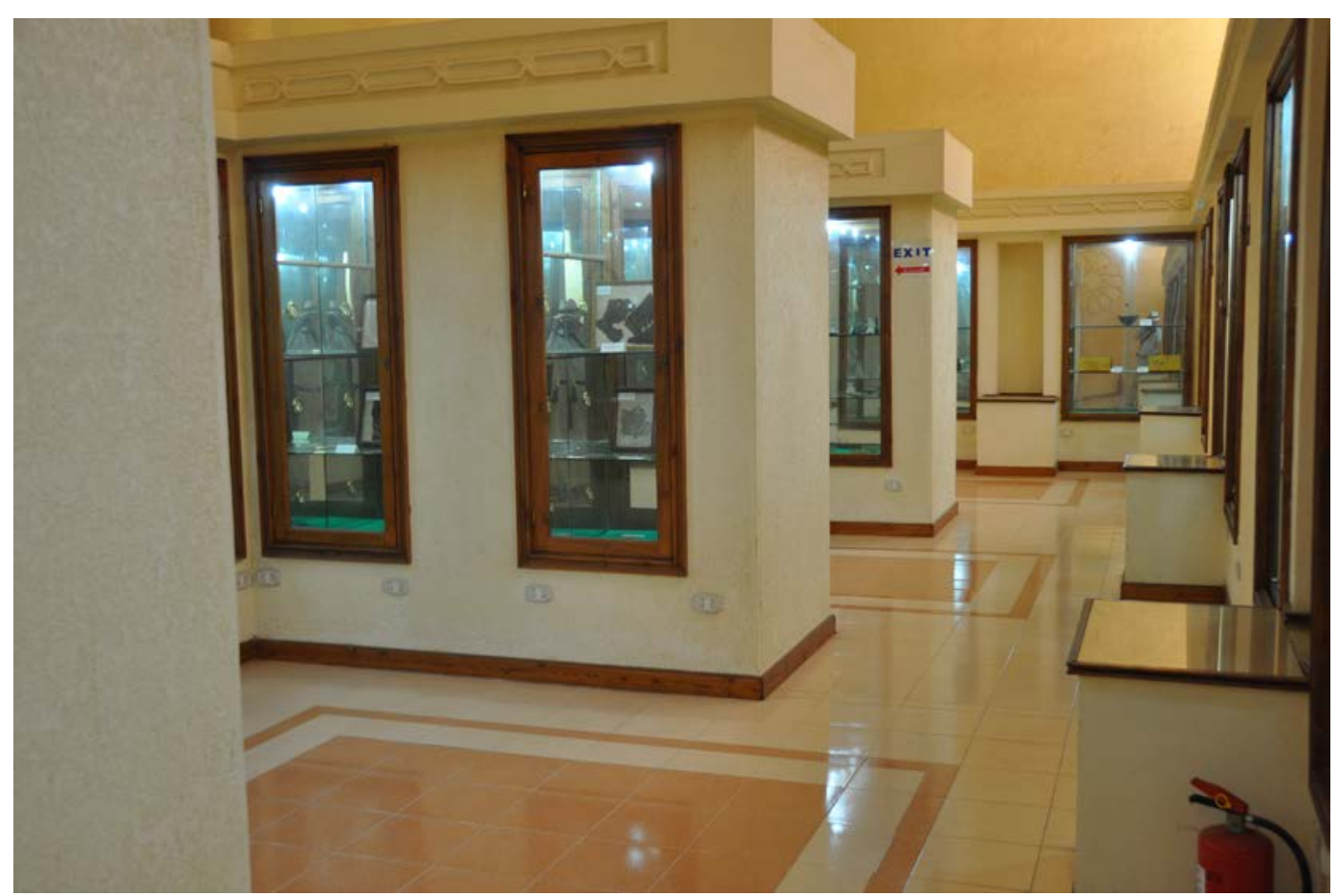

Salle du Musée de la Faculté des Lettres de l'Université d'Alexandrie (photo V. EUVERTE). 


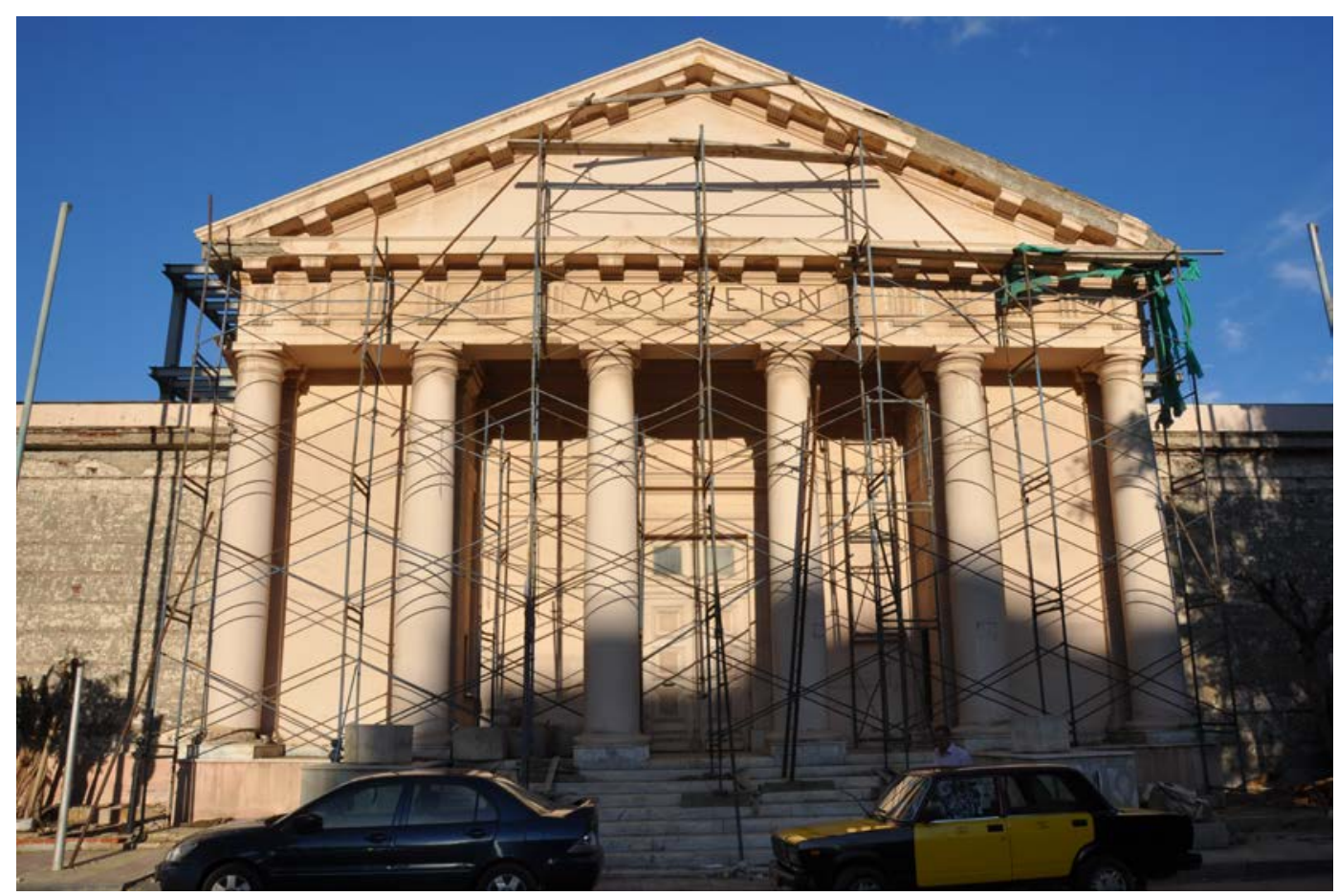

Façade du Musée gréco-romain d'Alexandrie (photo V. EUVERTE).

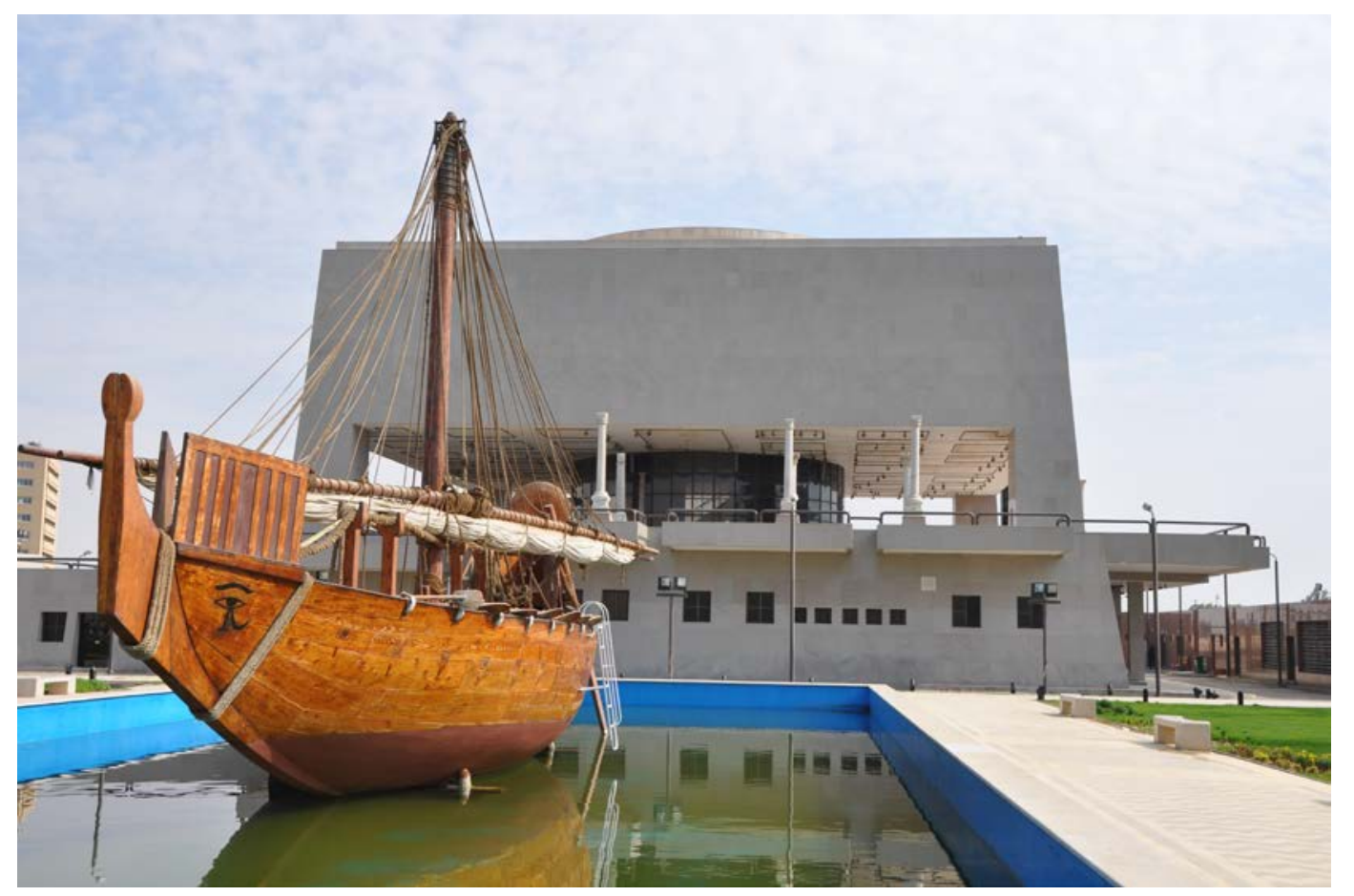

Musée de Suez (photo V. EUVERTE). 